\title{
Produção de snacks extrusados à base de polvilho doce e fibra de laranja
}

\author{
Production of extruded snacks from cassava starch and orange fiber
}

Magali Leonel ${ }^{\mathrm{I}}$ Luciana Bronzi de SouzaII Martha Maria Mischan'II

\section{RESUMO}

Este trabalho teve por objetivo avaliar o efeito da umidade, temperatura de extrusão e rotação da rosca na produção de produtos extrusados expandidos com a utilização do polvilho doce e da polpa de laranja desidratada como matéria-prima. O processo seguiu delineamento central composto rotacional com três fatores, totalizando 15 tratamentos (oito pontos fatoriais, seis pontos axiais e um ponto central com seis repetições). Os produtos obtidos foram caracterizados quanto ao índice de expansão (IE), ao volume específico (VE), ao índice de solubilidade em água (ISA) e à cor $\left(L^{*}, a^{*}\right.$ e $\left.b^{*}\right)$. Os resultados mostraram efeito das condições operacionais do processo sobre as características físicas dos produtos, e produtos claros com maior expansão e menor solubilidade em água foram obtidos nas condições mais elevadas de temperatura $\left(80-90^{\circ} \mathrm{C}\right)$, condições intermediárias de umidade (16\%) e rotação da rosca (218rpm).

Palavras-chave: amido, mandioca, polpa cítrica, extrusão, expansão, cor.

\section{ABSTRACT}

This research aimed to evaluate the effect of moisture, extrusion temperature and screw speed in the production of flax snacks using as raw material cassava starch and dehydrated orange pulp. The process followed the central composite rotational design to three factors, totaling 15 treatments (8 factorial points, 6 star points and 1 center point with 6 repetitions). The products obtained were characterized for expansion rate, specific volume, water solubility index and color $\left(L^{*}, a^{*}\right.$ and $\left.b^{*}\right)$. The results showed the effect of operational conditions on the physical characteristics of products, and clear products with larger expansion and lower water solubility index were obtained in conditions of the highest temperature (80$90^{\circ} \mathrm{C}$ ) and moisture (16\%) and intermediate screw speed (218rpm)

Key words: starch, cassava, citric pulp, extrusion, expansion, color.

\section{INTRODUÇÃO}

Com o aumento da expectativa de vida e a grande cobertura da mídia pelas questões da saúde, os consumidores estão mais interessados nos benefícios potenciais da nutrição para o controle e a prevenção de doenças e passaram a exigir em alimentos industrializados, além de sabor agradável e praticidade, alto valor nutritivo e outros benefícios à saúde (ARABBI, 2001; BOMBO, 2006).

O mercado de snacks no Brasil, especificamente o mercado de "salgadinhos", apresenta um faturamento anual de aproximadamente R\$ 1 bilhão, com produção em torno de 80 mil toneladas, apresentando crescimento das linhas de produtos com apelos nutricionais, como os fortificados, com baixo teor de gordura, e os funcionais (FERREIRA, 2006).

A extrusão é um processo contínuo, no qual a matéria-prima é forçada por meio de uma matriz ou de um molde, em condições de mistura e aquecimento, pressão e fricção, levando à ruptura de pontes de

ICentro de Raízes e Amidos Tropicais, Universidade Estadual Paulista "Júlio de Mesquita Filho" (UNESP), 18618-970, Botucatu, SP, Brasil. E-mail: mleonel@fca.unesp.br. Autor para correspondência.

IIPrograma de Pós-graduação em Saúde Coletiva, Faculdade de Medicina, UNESP, Botucatu, SP, Brasil.

IIIDepartamento de Bioestatística, Instituto de Biociências, UNESP, Botucatu, SP, Brasil. 
hidrogênio (gelatinização do amido) e desnaturação de proteínas (THAKUR \& SAXENA, 2000).

O controle do processo de extrusão permite a obtenção de produtos com características variadas, melhorando a eficiência e economia da operação. $\mathrm{O}$ controle das condições de extrusão, tais como temperatura, taxa de compressão da rosca, taxa de alimentação, teor de umidade e componentes de alimentação, é essencial para garantir a boa qualidade do produto e evitar perdas de nutrientes (CARVALHO et al., 2002).

No desenvolvimento de produtos extrusados expandidos, são parâmetros de qualidade desejados: elevados índices de expansão, baixa densidade, elevado volume específico, cor clara e elevada crocância.

Durante o processo de fabricação do suco de laranja, são gerados vários subprodutos. A polpa de laranja é composta pelas vesículas, que armazenam o suco, e pelas membranas, que separam as vesículas em gomos. De acordo com SOUZA et al. (2007), a polpa de laranja desidratada apresenta $85,31 \%$ de fibras totais, sendo $38,16 \%$ fibras solúveis e $47,15 \%$ fibras insolúveis.

Uma possibilidade de incremento na agroindustrialização da mandioca seria o uso de seus derivados, como o polvilho doce, em produtos de elevado valor agregado, como os extrusados funcionais. Nessa linha, este trabalho objetivou avaliar o efeito das condições operacionais do processo de extrusão, temperatura e velocidade da rosca, e da umidade de misturas de polvilho doce e polpa de laranja desidratada sobre as características físicas de produtos expandidos.

\section{MATERIAL E MÉTODOS}

No preparo das misturas de polvilho doce e polpa de laranja desidratada, o teor de fibras foi ajustado para $10 \%$ e, após a homogeneização em misturador elétrico, foram separadas 20 amostras de $800 \mathrm{~g}$ para o posterior condicionamento da amostra para os diferentes níveis de umidade.
A extrusão foi efetuada em uma linha completa de extrusão INBRA RX da Imbramaq S/A, com motor de 10HP acoplado a redutor de velocidade, sistema de extrusão por meio de fricção mecânica, rosca simples de extrusão, sistema de refrigeração hidráulica para controle de temperatura na camisa de extrusão, velocidade variável e capacidade de produção de $45 \mathrm{~kg} \mathrm{~h}^{-1}$.

$\mathrm{O}$ processo de extrusão teve como parâmetros fixos: taxa de compressão da rosca $(3: 1)$; comprimento da rosca $(45 \mathrm{~cm})$, diâmetro da rosca $(1,9 \mathrm{~cm})$; taxa de alimentação $\left(150 \mathrm{~g} \mathrm{~min}^{-1}\right)$; abertura da matriz $(4 \mathrm{~mm})$; temperatura fixa na $1^{\underline{a}} \mathrm{zona}\left(25^{\circ} \mathrm{C}\right)$ e na $2^{\mathrm{a}}$ zona $\left(40^{\circ} \mathrm{C}\right)$; e porcentagem de fibras na mistura $(10 \%$ na base úmida). Foram consideradas como variáveis independentes no processo: a temperatura de extrusão ( $3^{\mathrm{a}}$ zona), a rotação da rosca e a umidade da mistura. As variáveis dependentes foram: índice de expansão, volume específico $\left(\mathrm{mL} \mathrm{g}^{-1}\right)$, índice de solubilidade em água $(\%)$ e cor $\left(\mathrm{L}^{*}, \mathrm{a}^{*} \mathrm{e} \mathrm{b}^{*}\right)$.

Para analisar o efeito combinado das variáveis independentes nas características físicas dos extrusados, foi utilizado o delineamento central composto rotacional para três fatores, totalizando 15 tratamentos: oito tratamentos correspondentes ao fatorial $2^{3}$, em que os três fatores são: $\mathrm{T}=$ temperatura na $3^{\mathrm{a}}$ zona de aquecimento $\left({ }^{\circ} \mathrm{C}\right), \mathrm{U}=$ umidade da mistura (\%) e $\mathrm{R}=$ rotação da rosca (rpm), cada qual em dois níveis, codificados como $-1 \mathrm{e}+1$; seis tratamentos com os níveis mínimo e máximo de cada fator, codificados como $-\alpha$ e $+\alpha$, respectivamente, sendo $\alpha=23 / 4=1,682$; um tratamento central repetido seis vezes, em que os fatores estão todos em um nível médio, codificado como zero, a fim de estimar o erro experimental (Tabela 1).

$O$ índice de expansão dos extrusados foi avaliado no material após a extrusão e antes da secagem. Foi calculado pela relação entre o diâmetro da amostra e o diâmetro da matriz. O valor considerado foi obtido pela média aritmética das medidas de 20 diferentes produtos expandidos dentro de cada tratamento.

O volume específico dos produtos expandidos foi determinado pelo método do

Tabela 1- Parâmetros variáveis do processo de extrusão.

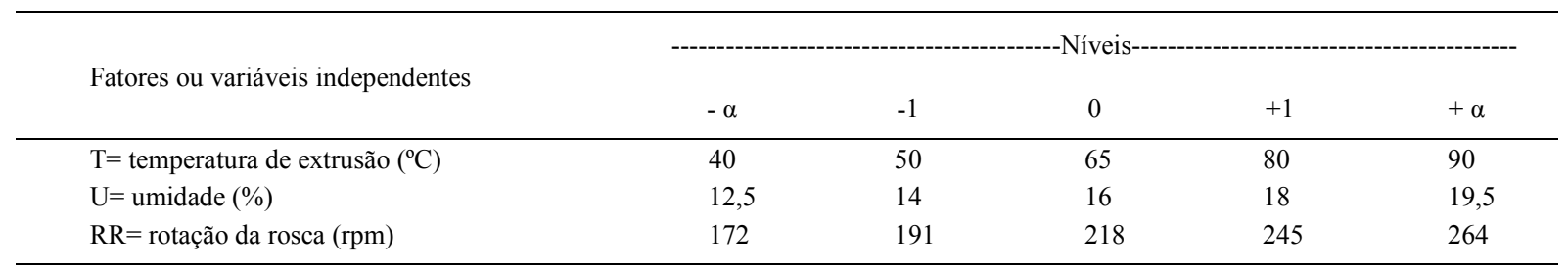

$\alpha=1,682$; Nível zero= seis repetições.

Ciência Rural, v.40, n.6, jun, 2010. 
deslocamento da massa ocupada (semente de painço) e determinado o seu volume em uma proveta graduada. Os resultados foram obtidos pela média aritmética das medidas de 20 diferentes produtos por tratamento e expressos em $\mathrm{mL} \mathrm{g}^{-1}$.

$O$ índice de solubilidade em água (ISA) foi determinado segundo ANDERSON et al. (1969), sendo os resultados expressos em porcentagem.

A cor dos produtos foi avaliada em colorímetro Minolta CR-400, com a utilização de iluminante D65, sendo feitas leituras de três amostras dos produtos extrusados moídos para cada tratamento. Os resultados foram expressos, no sistema CIELAB, em valores $L^{*}, a^{*}$ e $b^{*}$, em que os valores de $L^{*}$ (luminosidade ou brilho) variam do preto (0) ao branco (100), os valores do croma $a^{*}$ variam do verde (-60) ao vermelho $(+60)$ e os valores do croma $b^{*}$ variam do azul $(-60)$ ao amarelo $(+60)$.

$\mathrm{O}$ processamento dos dados e a análise estatística foram realizados com o auxílio do sistema SAS. A significância do modelo foi testada pela análise de variância (ANOVA), sendo adotado o nível de significância de $5 \%(\mathrm{P} \leq 0,05)$. Na análise dos dados realizada pelo programa SAS foi utilizada a análise de regressão com a opção stepwise. O modelo obtido foi validado pelo teste $\mathrm{F}$ utilizando-se como denominador o quadrado médio do erro puro. $\mathrm{Na}$ construção das superfícies de resposta, duas variáveis independentes variaram dentro das regiões estudas, e a terceira foi mantida fixa no ponto central.

\section{RESULTADOS E DISCUSSÃO}

A expansão do material ocorre na saída do extrusor em razão da diferença de temperatura e pressão interna e externa. Com o superaquecimento do produto, ocorre rápida descompressão, devido à evaporação da água na saída da matriz e à formação de células de ar.

Os valores obtidos para o índice de expansão dos produtos extrusados variaram de 1,93 a 2,99 (Tabela 2). A análise dos coeficientes de regressão mostrou ter ocorrido influência significativa da interação dos fatores umidade e rotação sobre o índice de expansão (IE) dos extrusados (Tabela 3).

HASHIMOTO \& GROSSMANN (2003), analisando o efeito das condições de extrusão sobre a qualidade de extrusados de misturas de farelo e amido de mandioca, obtiveram índice de expansão variando de 1,6 a 3,2, valores próximos aos obtidos neste trabalho.

Tabela 2 - Médias dos dados experimentais de índice de expansão (IE), volume específico (VE), índice de solubilidade em água (ISA) e cor (L*, a* e b*) dos produtos extrusados nas diferentes condições experimentais.

\begin{tabular}{|c|c|c|c|c|c|c|c|c|c|}
\hline & $\mathrm{T}$ & $\mathrm{U}$ & RR & IE & VE & ISA & $\mathrm{L}^{*}$ & $a^{*}$ & $b^{*}$ \\
\hline 1 & 50 & 14 & 190 & 2,94 & 3,68 & 53,15 & 79,07 & 2,85 & 20,95 \\
\hline 2 & 80 & 14 & 190 & 2,82 & 6,32 & 71,74 & 79,56 & 3,16 & 20,07 \\
\hline 3 & 50 & 18 & 190 & 2,81 & 2,21 & 49,82 & 79,28 & 2,53 & 21,97 \\
\hline 4 & 80 & 18 & 190 & 2,45 & 7,15 & 52,94 & 79,78 & 3,29 & 18,69 \\
\hline 5 & 50 & 14 & 245 & 2,86 & 4,85 & 35,88 & 76,85 & 3,77 & 22,25 \\
\hline 6 & 80 & 14 & 245 & 1,93 & 6,70 & 66,38 & 68,25 & 5,42 & 21,37 \\
\hline 7 & 50 & 18 & 245 & 2,81 & 4,10 & 58,18 & 79,25 & 3,02 & 19,89 \\
\hline 8 & 80 & 18 & 245 & 2,79 & 5,21 & 73,38 & 76,91 & 3,78 & 23,64 \\
\hline 9 & 65 & 16 & 218 & 2,76 & 3,82 & 51,66 & 77,27 & 3,22 & 20,42 \\
\hline 10 & 65 & 16 & 218 & 2,75 & 3,75 & 51,76 & 77,17 & 3,37 & 20,97 \\
\hline 11 & 65 & 16 & 218 & 2,71 & 3,75 & 51,44 & 77,18 & 3,57 & 20,40 \\
\hline 12 & 65 & 16 & 218 & 2,71 & 3,94 & 51,08 & 77,14 & 3,32 & 20,60 \\
\hline 13 & 65 & 16 & 218 & 2,70 & 3,83 & 51,69 & 77,07 & 3,49 & 20,63 \\
\hline 14 & 65 & 16 & 218 & 2,71 & 3,82 & 51,60 & 77,11 & 3,67 & 20,26 \\
\hline 15 & 40 & 16 & 218 & 2,50 & 1,87 & 43,63 & 77,48 & 2,52 & 19,80 \\
\hline 16 & 90 & 16 & 218 & 2,52 & 5,50 & 45,26 & 77,19 & 4,14 & 21,91 \\
\hline 17 & 65 & 12 & 218 & 2,24 & 8,44 & 70,04 & 70,06 & 5,54 & 23,16 \\
\hline 18 & 65 & 20 & 218 & 2,66 & 3,36 & 84,93 & 79,37 & 3,06 & 22,07 \\
\hline 19 & 65 & 16 & 163 & 2,99 & 5,17 & 63,04 & 79,00 & 3,55 & 21,69 \\
\hline 20 & 65 & 16 & 272 & 2,47 & 6,74 & 37,76 & 77,06 & 3,79 & 19,84 \\
\hline
\end{tabular}

$\mathrm{T}=$ temperatura de extrusão $\left({ }^{\circ} \mathrm{C}\right), \mathrm{U}=$ umidade das misturas $(\%), \mathrm{RR}=$ rotação da rosca (rpm)

Ciência Rural, v.40, n.6, jun, 2010. 
Tabela 3- Coeficientes de regressão estimados (modelo $\mathrm{y}_{\mathrm{k}}=\beta_{0}+\beta_{1} \mathrm{x}_{1}+\beta_{2} \mathrm{x}_{2}+\beta_{12} \mathrm{x}_{1} \mathrm{x}_{2}$ ).

\begin{tabular}{|c|c|c|c|c|c|c|}
\hline \multirow{2}{*}{ Parâmetros } & \multicolumn{6}{|c|}{ - } \\
\hline & IE & VE & ISA & $\mathrm{L}^{*}$ & $\mathrm{a}^{*}$ & $b^{*}$ \\
\hline$\beta_{0}$ (intercepto) & 2,72 & 3,82 & 51,54 & 77,13 & 3,45 & 20,56 \\
\hline$\beta_{1}(\mathrm{~T})$ & $-0,10$ & $1,22 * * *$ & $5,14^{*}$ & $-0,76$ & $0,45^{* *}$ & 0,16 \\
\hline$\beta_{2}(\mathrm{U})$ & 0,074 & $-0,84 * * *$ & 2,36 & $1,99 * * *$ & $-0,49 * * *$ & $-0,17$ \\
\hline$\beta_{3}(\mathrm{RR})$ & $-0,11$ & 0,30 & $-2,66$ & $-1,44 * *$ & $0,33 * *$ & 0,173 \\
\hline$\beta_{11}\left(\mathrm{~T}^{2}\right)$ & $-0,049$ & $-0,097$ & $-2,53$ & 0,21 & $-0,104$ & 0,03 \\
\hline$\beta_{22}\left(\mathrm{U}^{2}\right)$ & $-0,071$ & $0,69 * *$ & $9,15 * * *$ & $-0,72$ & $0,24 *$ & $0,65 * *$ \\
\hline$\beta_{33}\left(\mathrm{RR}^{2}\right)$ & 0,028 & $0,71 * *$ & $-0,43$ & 0,46 & 0,02 & 0,001 \\
\hline$\beta_{12}\left(\mathrm{~T}^{*} \mathrm{U}\right)$ & 0,084 & 0,195 & $-3,85$ & 0,78 & $-0,05$ & 0,28 \\
\hline$\beta_{13}\left(\mathrm{~T}^{*} \mathrm{RR}\right)$ & $-0,0587$ & $-0,58^{*}$ & 3,00 & $-1,49^{*}$ & 0,17 & $0,88^{*}$ \\
\hline$\beta_{23}\left(\mathrm{U}^{*} \mathrm{RR}\right)$ & $0,1637^{*}$ & $-0,20$ & $6,43 *$ & $1,33^{*}$ & $-0,27$ & 0,034 \\
\hline $\mathrm{R}^{2}$ & 0,6828 & 0,8855 & 0,8366 & 0,8529 & 0,8337 & 0,4997 \\
\hline $\mathrm{CV}$ & 0,94 & 1,48 & 0,45 & 0,09 & 4,71 & 1,18 \\
\hline F valor & 2,39 & 8,59 & 5,69 & 6,45 & 5,57 & 1,11 \\
\hline $\operatorname{Pr}>F$ & 0,0952 & 0,0012 & 0,0060 & 0,0037 & 0,0065 & 0,4333 \\
\hline
\end{tabular}

$\beta_{1}(\mathrm{~T})=$ temperatura (linear) ; $\beta_{2}(\mathrm{U})=$ umidade (linear); $\beta_{3}(\mathrm{RR})=$ rotação da rosca (linear); $\beta_{11}\left(\mathrm{~T}^{2}\right)=$ temperatura $($ quadrático $) ; \beta_{22}\left(\mathrm{U}^{2}\right)=$ umidade (quadrático); $\beta_{33}\left(\mathrm{RR}^{2}\right)=$ rotação da rosca (quadrático); $\mathrm{R}^{2}=$ coeficiente de determinação; CV (\%) coeficiente de variação em porcentagem; $*=\mathrm{P}<0.05, * *=\mathrm{P}<0.01, * * *=\mathrm{P}<0.001 ; \mathrm{IE}=$ índice de expansão; $\mathrm{VE}=$ volume específico $\left(\mathrm{mL} \mathrm{g}^{-1}\right)$; ISA=índice de solubilidade em água $(\%)$; $\mathrm{L}^{*}=$ luminosidade; $\mathrm{a} *=$ croma $\mathrm{a} ; \mathrm{b} *=$ croma $\mathrm{b}$.

O volume específico (VE) é uma medida da expansão volumétrica, que é a soma das expansões radial e axial. Os valores de VE variaram de 1,87 a 8,44ml $\mathrm{g}^{-1}$ (Tabela 2). O maior valor experimental observado para o volume específico dos snacks está próximo ao relatado por ALVES \& GROSSMANN (2002), para snacks comerciais de milho $\left(8,72 \mathrm{~mL} \mathrm{~g}^{-1}\right)$.

O modelo de regressão foi significativo para o VE, sendo observado efeito da temperatura, umidade e rotação da rosca sobre essa característica (Tabela 3 ). As superfícies de resposta mostram que foram obtidos snacks com maior volume específico nas condições de baixa umidade e elevada temperatura de extrusão e de baixa rotação da rosca e elevada temperatura (Figura 1).

De acordo com MERCIER et al. (1998), a expansão aumenta com a elevação da temperatura, quando o conteúdo de umidade do material fica ao redor de $19 \%$, devido à menor viscosidade, permitindo expandir mais rápido a massa fundida, ou devido ao aumento na pressão de vapor. Em temperaturas baixas de extrusão, a expansão diminui porque o amido não é completamente fundido. O grau de expansão radial é proporcional à temperatura até certo valor, para depois decrescer em temperaturas elevadas. A diminuição da expansão a temperaturas muito altas é atribuída ao aumento da dextrinização e enfraquecimento da estrutura.
CAMARGO et al. (2008), avaliando as propriedades físicas de biscoitos extrusados de polvilho azedo com fibras, observaram variação de volume específico de $1,5 \mathrm{~mL} \mathrm{~g}^{-1}$ a $5,6 \mathrm{~mL} \mathrm{~g}^{-1}$, com efeito significativo da umidade e da porcentagem de fibras. Nas condições de elevado teor de fibras $(7,5 \%)$, o volume específico foi maior com a umidade elevada $(20 \%)$.

O aumento da solubilidade em produtos extrusados é atribuído à dispersão das moléculas de amilose e de amilopectina em consequência da gelatinização, em condições brandas, e da formação de compostos de baixo peso molecular, em condições severas (COLONNA et al., 1984).

Os resultados mostraram influência significativa dos fatores: temperatura de extrusão (efeito linear), umidade da mistura (efeito quadrático) e interação umidade e rotação da rosca sobre o índice de solubilidade em água dos produtos extrusados (Tabela 3). O índice de solubilidade em água dos produtos extrusados variou de 37,76 a 84,93\% (Tabela 2), com os menores valores observados nas condições de baixa temperatura, com umidade e rotação da rosca nas condições intermediárias (Figura 1).

A luminosidade das amostras extrusadas variou de 68,25 a 79,78 (Tabela 2), indicando interferência significativa de umidade e rotação da rosca (efeitos lineares) e de interação da rotação da rosca 


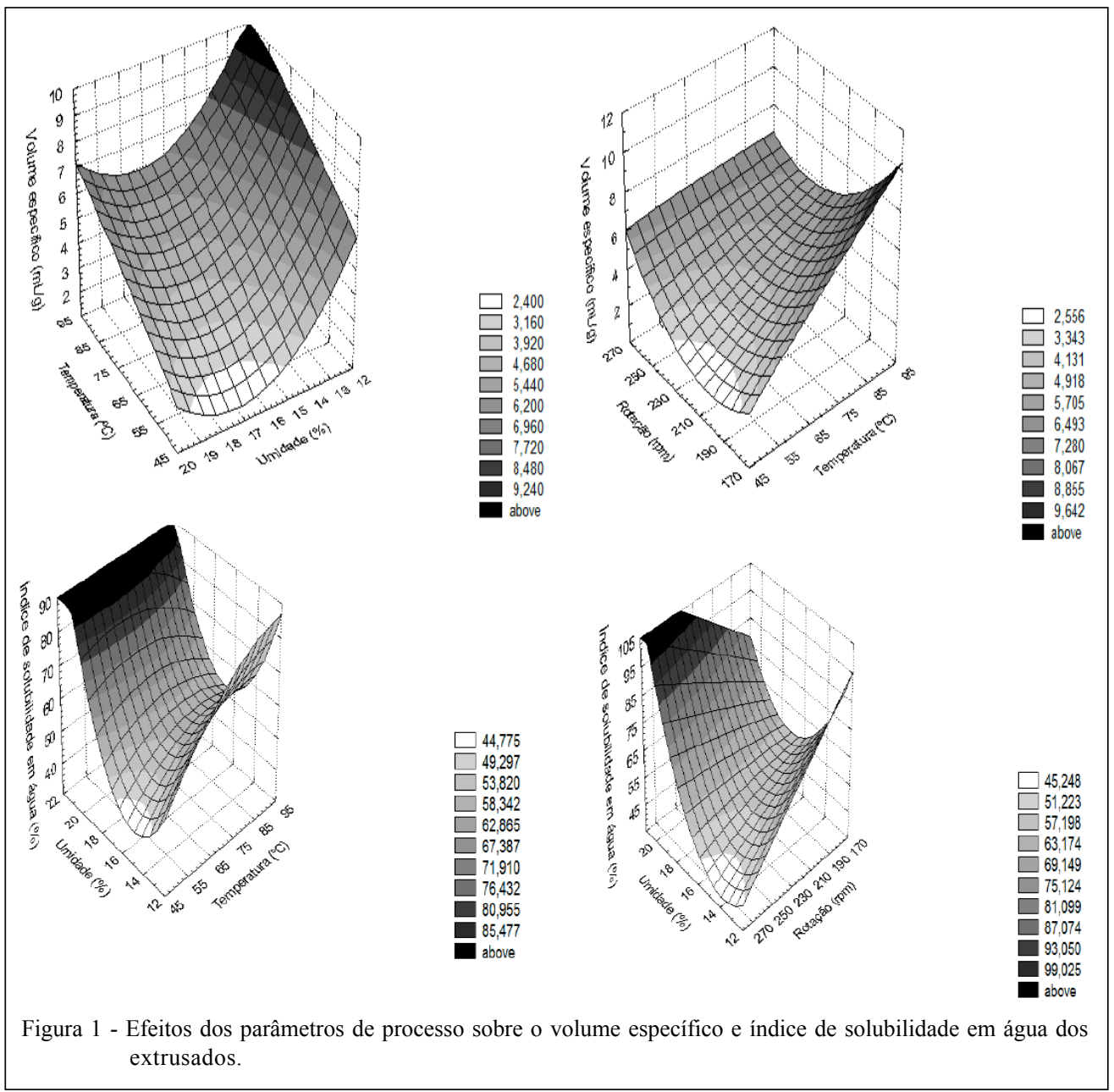

com a temperatura e com a umidade na claridade das amostras (Tabela 3 ).

As condições de baixa umidade e alta rotação foram as de menor luminosidade das amostras, enquanto que, nas condições de elevada temperatura e rotação, foi evidenciada a influência das condições severas de processamento no escurecimento dos produtos (Figura 2).

O valor de $\mathrm{a}^{*}$ variou de 2,52 a 5,54, indicando a presença de vermelho em todas as amostras (Tabela 2). A análise dos coeficientes de regressão mostrou efeito significativo dos três fatores do modelo: temperatura (efeito linear), umidade (efeitos linear e quadrático) e rotação da rosca (efeito linear), sobre esse componente de cromaticidade (Tabela 3).

Os menores valores de croma $a^{*}$ são observados nas condições de baixa rotação e temperatura de extrusão. Nas condições de elevada rotação e temperatura, com baixa umidade, os valores a* são maiores (Figura 2).
MENEGAZZI et al. (2007), avaliando os efeitos de parâmetros de extrusão sobre a cor de produtos extrusados de mandioquinha-salsa, observaram aumento dos valores de croma $a^{*}$ e $b^{*}$ com o processo de extrusão e efeito significativo da umidade sobre o croma $\mathrm{a}^{*} \mathrm{e}$ da temperatura de extrusão sobre o croma $b^{*}$. Condições intermediárias de umidade $(15 \%)$, temperatura $\left(120^{\circ} \mathrm{C}\right)$ e rotação da rosca $(204 \mathrm{rpm})$ promoveram a obtenção de produtos mais claros.

A variação do componente de cromaticidade $b^{*}$ foi de 18,69 a 23,16, mostrando forte presença de amarelo nas amostras, contudo com baixa interferência dos tratamentos sobre esse parâmetro (Tabela 2). A análise dos coeficientes de regressão mostrou efeito significativo da umidade (efeito quadrático) e da interação da temperatura e rotação da rosca sobre o croma b* (Tabela 3).

Segundo BADRIE \& MELLOWES (1991), a elevação da temperatura aumenta a intensidade da cor, e os altos níveis de umidade resultam em produtos 


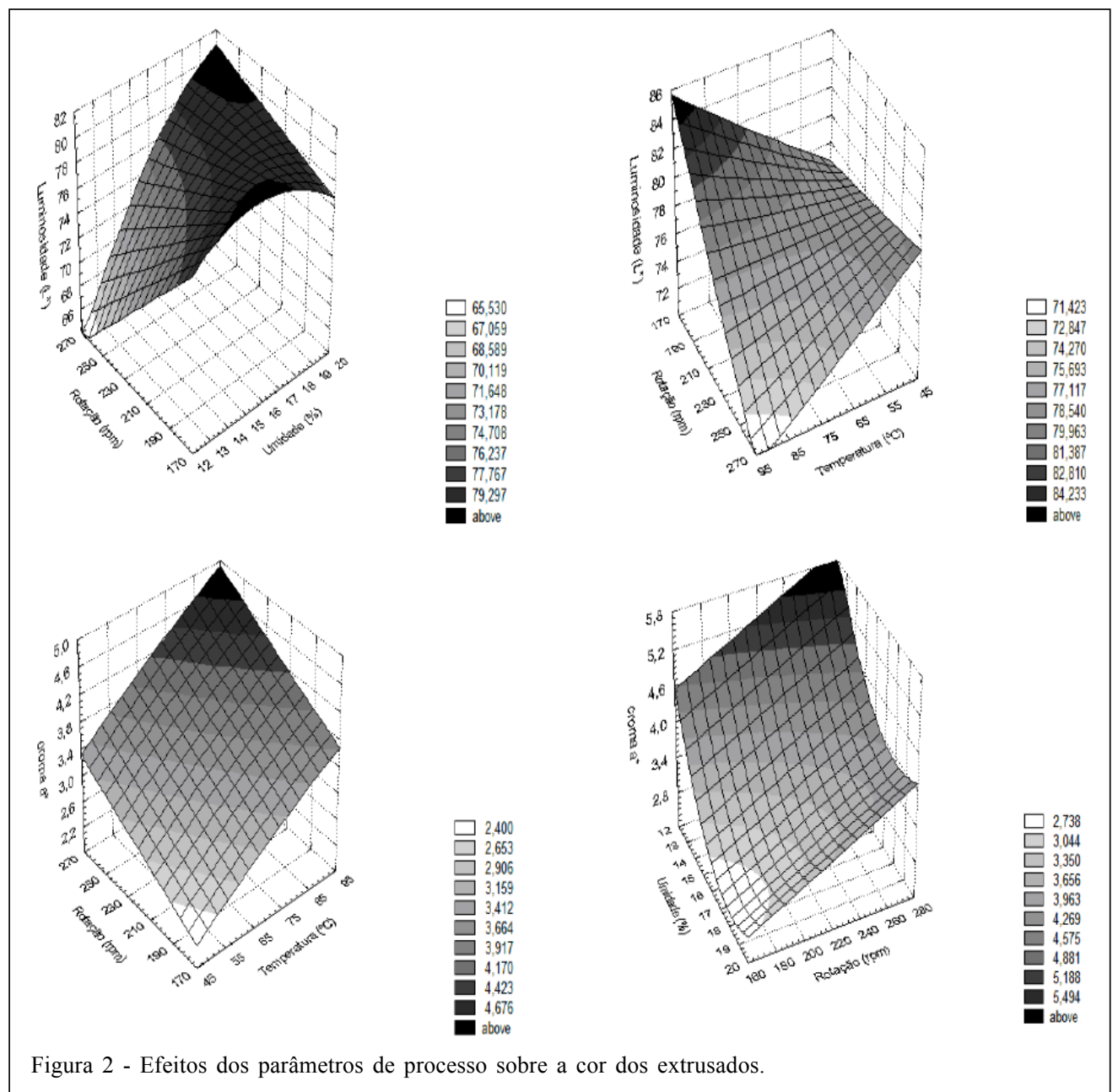

mais claros, pois o aumento da umidade reduz o tempo de residência, proporcionando menor ocorrência de caramelização e reação de Maillard.

\section{CONCLUSÃO}

Nas condições testadas neste trabalho, os parâmetros do processo de extrusão: umidade da mistura, temperatura de extrusão e rotação da rosca interferiram significativamente nas propriedades físicas dos produtos expandidos. Entre os parâmetros estudados, a umidade teve efeito sobre todas as propriedades físicas, seguida pela temperatura de extrusão, que teve efeito linear sobre o volume específico, índice de solubilidade e croma a*, e a rotação da rosca, que afetou principalmente a cor dos produtos extrusados.

Produtos claros, com elevada expansão e volume específico e menor solubilidade em água, foram obtidos nas condições de elevada temperatura de extrusão, com rotação da rosca e umidade das misturas nos níveis médios testados.

\section{REFERÊNCIAS}

ALVES, R.M.L., GROSSMANN, M.V.E. Parâmetros de extrusão para produção de "snacks" de farinha de cará (Dioscorea alata). Ciência e Tecnologia de Alimentos, Campinas, v.22, n.1, p.32-38, 2002. Disponível em: <http://www.scielo.br/ scielo.php? script=sci_arttext\&pid=S0101$20612002000100006 \& \operatorname{lng}=\mathrm{en} \& \mathrm{nrm}=\mathrm{iso}>$. Acesso em: 10 maio, 2010. doi: 10.1590/S0101-20612002000100006.

ANDERSON, R.A. et al. Gelatinization of corn grits by roll and extrusion cooking. Cereal Science Today, St Paul, v.14, n.1, p.4-11, 1969.

ARABBI, P.R. Alimentos funcionais - Aspectos gerais. Nutrire: Revista da Sociedade Brasileira de Alimentação e Nutrição, São Paulo, v.21, p.87-102, 2001. Disponível em: $<$ http://www.sban.com.br/educacao/nutrire/21/nut21_7.htm>. Acesso em: 07 abr. 2010.

Ciência Rural, v.40, n.6, jun, 2010. 
BADRIE, N.; MELLOWES, W.A. Effect of extrusion variables on cassava extrudates. Journal of Food Science, Chicago, v.56, p.1334-1337, 1991. Disponível em: <http:// www3.interscience.wiley.com/journal/119344950/issue $>$. Acesso em: 07 abr. 2010

BOMBO, A.J. Obtenção e caracterização nutricional de snacks de milho (Zea mays L.) e linhaça (Linum usitatissimum L.). 2006. 96f. Dissertação (Mestrado em Saúde Pública) - Faculdade de Saúde Pública, Universidade de São Paulo, SP.

CAMARGO, K.F. et al. Produção de biscoitos extrusados de polvilho azedo com fibras: efeito de parâmetros operacionais sobre as propriedades físicas. Ciência e Tecnologia de Alimentos, Campinas, v.28, n.3, p.586-591, 2008. Disponível em: <http://www.scielo.br/pdf/cta/v28n3/a13v28n3.pdf>. Acesso em: 05 abr. 2010.

CARVALHO, R. V. et al. Efeito dos parâmetros de extrusão nas propriedades físicas de extrusados $(3 \mathrm{G})$ de misturas de farinhas de trigo, arroz e banana. Ciência e Agrotecnologia, Lavras, v.26, n.5, p.1006-1018, 2002. Disponível em: <http:// www.editora.ufla.br/revista/26 5/art17.PDF>. Acesso em: 07 abr. 2010 .

COLONNA, P. et al. Extrusion cooking and drum drying of wheat starch. I. physical and macromolecular modifications. Cereal Chemistry, St Paul, v.61, n.6, p.538-543, 1984

FERREIRA, R.E. Avaliação de parâmetros do processo de extrusão e do teor de farelo de trigo adicionado em características de "snacks" expandidos de milho. 2006. 162f. Tese (Doutorado em Tecnologia de Alimentos) - Faculdade de Engenharia de Alimentos, Universdiade Estadual de Campinas, SP.
HASHIMOTO, J.M.; GROSSMANN, M.V.E. Effects of extrusion conditions on quality of cassava bran/cassava starch extrudates. International Journal of Food Science \& Technology, Oxford, v.38, n.5, p.511-517, 2003. Disponível em: <http://www3.interscience.wiley.com/cgi-bin/fulltext/ 118888399/PDFSTART>. Acesso em: 10 maio, 2010.

MENEGASSI, B. et al. Efeito de parâmetros de extrusão na cor e propriedades de pasta de farinha de mandioquinha-salsa (Arracacia xanthorrhiza). Ciência e Agrotecnologia, Lavras, v.31, n.6, p.1780-1792, 2007. Disponível em: <http:/ /www.scielo.br/pdf/cagro/v31n6/a27v31n6.pdf>. Acesso em: 05 abr. 2010 .

MERCIER, C. et al. Extrusion cooking. 2.ed. St. Paul: American Association of Cereal Chemists, 1998. 471p.

SOUZA, L.B. et al . Efeitos dos parâmetros de extrusão nas propriedades físicas de extrusados de misturas de fécula de mandioca e polpa cítrica. Alimentos e Nutrição, Araraquara v.18, n.1, p.83-91, 2007. Disponível em: <http:// 200.145.71.150/seer/index.php/alimentos/article/view/138/ 147>. Acesso em: 05 abr. 2010 .

THAKUR, S.; SAXENA, D.C. Formulation of extruded snack food (gum based cereal-pulse blend): optimization of ingredients levels using response surface methodology. Lebensmittel-Wissenschaft und-Technologie, v.33, p.354-361, 2000. Disponível em: <http:/ /www.sciencedirect.com/science? ob=MImg\& imagekey=B6WMV45 F 4 KP8-19-1\&_cdi $=6944 \&$ \& ser $=972052 \&$ \&ii $=$ S 0 $023643800906686 \&$ orig $=$ search\& coverD ate $=08 \% 2 \mathrm{~F} 31 \% 2 \mathrm{~F} 2000 \&$ sk $=999669994 \& v i e w=\mathrm{c} \& w \mathrm{wp}=\mathrm{dGLbVzW}$ zSkzS\&md5=f17794a 9 fa98d74981 ec73cd03bdbb62\&ie=/ sdarticle.pdf $>$. Acesso em: 10 maio, 2010. doi:10.1006/ fst1.2000.0668. 